\title{
Genetic variability and discrimination of low doses of Toxocara spp. from public areas soil inferred by loop-mediated isothermal amplification assay as a field-friendly molecular tool
}

\author{
Maryam Ozlati ${ }^{1,2}$, Adel Spotin ${ }^{1,2}$, Abbas Shahbazi², Mahmoud Mahami-Oskouei², Teimour Hazratian², Mohammad Adibpor ${ }^{2}$ \\ Ehsan Ahmadpour ${ }^{3}$, Afsaneh Dolatkhah² and Paria Khoshakhlagh ${ }^{2}$ \\ 1. Infectious and Tropical Diseases Research Center, Tabriz University of Medical Sciences, Tabriz, Iran; 2. Department \\ of Parasitology and Mycology, Faculty of Medicine, Tabriz University of Medical Sciences, Tabriz, Iran; 3. Drug Applied \\ Research Center, Tabriz University of Medical Sciences, Tabriz, Iran. \\ Corresponding author: Adel Spotin, e-mail: adelespotin@gmail.com, \\ MO: maryamomedicals@gmail.com, AS: shahbazy42@yahoo.com, MMO:mmahami@gmail.com, \\ TH: hazratian2222@yahoo.com, MA: adibpourm@yahoo.com, EA: ehsanahmadpour@gmail.com, \\ AD: afsaneh.dolatkhah@gmail.com,PK: paria.khoshakhlagh@gmail.com \\ Received: 31-08-2016, Accepted: 24-11-2016, Published online: 27-12-2016
}

doi: 10.14202/vetworld.2016.1471-1477 How to cite this article: Ozlati M, Spotin A, Shahbazi A, Mahami-Oskouei M, Hazratian T, Adibpor M, Ahmadpour E, Dolatkhah A, Khoshakhlagh P (2016) Genetic variability and discrimination of low doses of Toxocara spp. from public areas soil inferred by loop-mediated isothermal amplification assay as a field-friendly molecular tool, Veterinary World, 9(12): 1471-1477.

\begin{abstract}
Aim: One of the main diagnostic problems of conventional polymerase chain reaction (PCR) is indiscrimination of low parasitic loads in soil samples. The aim of this study is to determine the genetic diversity and identification of Toxocara spp. from public areas soil inferred by loop-mediated isothermal amplification (LAMP) assay.

Materials and Methods: A total of 180 soil samples were collected from various streets and public parks of northwest Iran. The DNA of recovered Toxocara eggs were extracted and amplified by PCR and LAMP following $\mathrm{ZnSO}_{4}$ flotation technique. The amplicons of internal transcribed spacer-2 gene were sequenced to reveal the heterogeneity traits of Toxocara spp. In addition, Toxocara canis sequences of southwest Iran were directly retrieved to compare gene flow between two distinct populations.
\end{abstract}

Results: Toxocara spp. eggs were found in 57, 14 and 77 of soil samples using the microscopy, PCR and LAMP (detection limit 1-3 eggs/200 g soil), respectively. $7.7 \%$ of isolates were identified as T. canis by PCR method, while LAMP was able to detect $27.2 \%, 15.5 \%$ and $12.2 \%$ as Toxocara cati, T. canis and mixed infections, respectively. The kappa coefficient between LAMP and microscopy indicated a strong agreement (0.765) but indicated a faint agreement among LAMP-PCR (0.203) and PCR-microscopy $(0.308)$ methods. A pairwise fixation index $(F$ st) as a degree of gene flow was generally low $(0.02156)$ among Toxocara populations of northwest and southwest Iran.

Conclusions: The statistically significant $F$ st value indicates that the $T$. canis populations are not genetically well differentiated between northwest and southwest Iran. This shows that here is possibly an epidemiological drift due to the transfer of alleles. The LAMP assay because of its shorter reaction time, more sensitivity, and simultaneous detection of environmental contamination to be appears as valuable field diagnosis compared to PCR. Therefore, the detection of low Toxocara spp. loads from public area soils will help to expand epidemiological understanding of toxocariasis and establishing preventive strategies in resource-limited endemic of Iran.

Keywords: gene flow, Iran, loop-mediated isothermal amplification, polymerase chain reaction, soil, Toxocara spp.

\section{Introduction}

Human toxocariasis is a helminthic sapro-zoonotic disease that is caused by the larval stages of common gastrointestinal parasites of dogs (Toxocara canis) and cats (Toxocara cati) [1,2]. These animals play a notable role in the transmission of toxocariasis, particularly in the tropical, subtropical and temperate regions of the world [3]. Based on host immunity response against parasite and localization of the

Copyright: Ozlati, et al. Open Access. This article is distributed under the terms of the Creative Commons Attribution 4.0 International License (http://creativecommons.org/licenses/by/4.0/), which permits unrestricted use, distribution, and reproduction in any medium, provided you give appropriate credit to the original author(s) and the source, provide a link to the Creative Commons license, and indicate if changes were made. The Creative Commons Public Domain Dedication waiver (http://creativecommons.org/ publicdomain/zero/1.0/) applies to the data made available in this article, unless otherwise stated. larvae, four clinical appearances including visceral larva migrans, ocular larva migrans (OLM), neurological toxocariasis, and covert toxocariasis have been defined [3-6]. Human infections, especially children, are frequently acquired using environment contaminated with embryonated Toxocara spp. eggs by playing in public areas such as parks, sandpits, and playgrounds [7]. The increasing number of infected stray dogs and cats in public areas, their easy access to unfenced public parks and increasing popularity of keeping animals as pets have caused that rate of sero-prevalence of toxocariasis is unambiguously increasing among healthy children [7]. It is noteworthy that a large number of Toxocara eggs in the soil is unusual in sample collected from public places. Even in shelters for dogs and cats, some authors detected up to 10 eggs/50 g sample [8]. 
Given that the low numbers of eggs in children may cause OLM [9] employing validated diagnostic test should be broadly noticed among neglected hyperendemic foci. One of the principal diagnostic difficulties of single round polymerase chain reaction (PCR) concerning detection of Toxocara eggs in soil sources is associated with the presence of inhibitors such as humic acids, lipids, polysaccharides and polyphenols/tannins, which are strongly affected on extracted DNA yield. The accurate identification of $T$. canis and T. cati eggs owing to their similar morphology can be difficult to discriminate [10]. A number of PCR-based molecular assays (PCR, PCR-restriction fragment length polymorphism, and real-time PCR) have been conducted for differentiation of $T$. canis and T. cati eggs from parks, playgrounds, sandpits, backyards, farmyards gardens, and beaches [11-13]. However, the PCR assays take several hours and necessitate specialized equipment, which make their use impossible in under field conditions. Loop-mediated isothermal amplification (LAMP) has been recently introduced as a rapid and sensitive alternative diagnostic technique in resource-limited endemic regions. The extensive applicability of LAMP has established in the revealing of low parasitic loads in various samples such as Taenia spp. eggs in feces samples (detection limit 5 eggs/g of feces) Schistosoma japonicum in snails, Entamoeba histolytica trophozoites in feces samples (detection limit 1 trophozoite in $500 \mathrm{ml}$ of feces), Toxoplasma gondii in water samples, Leishmania, Plasmodium, and Dirofilaria immitis [14-20]. Up to now, only one report is available on the using the LAMP method for detection and discrimination of T. canis and T. cati eggs from sand samples [21]. The significant prevalence of latent and asymptomatic Toxocara infection among young population has identified in northwest Iran; however, there are not many molecular explorations on identification of Toxocara spp. in soil samples of Iran [22]. On the one hand, computations of gene flow index among different populations of Toxocara spp. could provide a worthwhile data concerning population genetic structures, epidemiological drift of parasite, allele frequencies and speciation.

The aim of this study was to appraise the soil contamination of public areas by Toxocara spp. eggs, in the northwest Iran inferred by microscopy, PCR, LAMP and phylogenetic strategies to identify the accurate prevalence rate, epidemiological drift and genetic variability and precise taxonomic status of parasite in the region.

\section{Materials and Methods}

Ethical approval

This research was approved by the Faculty of Medicine, Tabriz University of Medical Sciences, Tabriz, Iran.

\section{Survey area, collection and recovery of soil sample}

This examination was conducted from February 2014 to May 2015 in Tabriz city located at Northwest
Iran. Tabriz as the second largest city in Iran with $1,800,000$ populations has a cold to semi-arid climate with regular seasons. The average annual rainfall and temperature are $280 \mathrm{~mm}$ and $12.6^{\circ} \mathrm{C}$, respectively. A total of 180 soil samples were collected from five divisions (north, south, east, west and center) of the city covering public parks and streets. Each sample (approximately $200 \mathrm{~g}$ soil) was collected from $3 \mathrm{~cm}$ depth of soil and placed in labeled polyethylene bags.

\section{Microscopic observation and floatation technique}

After 1-2 days, the dried soil samples were sieved through a $0.5 \mathrm{~mm}$ mesh. To recovery of Toxocara eggs from contaminated soils, the zinc sulfate $\left(\mathrm{ZnSO}_{4}\right)$ flotation was employed according to modified Dada method [23]. In brief, $10 \mathrm{~g}$ samples with $50 \mathrm{ml}$ of $0.1 \%$ Tween 80 were vortexed for $30 \mathrm{~min}$. The supernatant free of eggs was decanted and a saturated flotation solution $\left(\mathrm{ZnSO}_{4}, 1.52\right.$ specific gravity) was added to the tubes containing the remaining sediment and centrifuged at $1500 \mathrm{rpm}$ for $10 \mathrm{~min}$. The solution was added to form a meniscus and a cover slip was overlaid. After $15 \mathrm{~min}$, the cover slip was transferred to a glass slide and was examined at a magnification of $40 \times$ and $100 \times$ for Toxocara eggs based on morphological characters such as presence of larva inside the pitted eggs. Furthermore, unembryonated eggs were incubated in $0.5 \%$ formalin at $25^{\circ} \mathrm{C}$ for more than 2 months.

\section{DNA extraction and PCR amplification}

The total DNA from all flotation fluid was extracted using the Takapozist DNA extraction kit according to the manufacturer's instructions. First, eggs were sonicated for 5 times in $20 \mathrm{~s}$. The homogenized samples were subjected through the freeze-thawing technique (10 cycles of freezing [10 min at liquid nitrogen] and thawing [10 $\mathrm{min}$ at $\left.95^{\circ} \mathrm{C}\right]$ ) and proteinase $\mathrm{K}$ digestion was performed overnight. To identify Toxocara spp. the species-specific primers were chosen from internal transcribed spacer 2 (ITS2) gene that were previously described as Tcan1 (5'-AGTATGATGGGCGCGCCAAT-3') and NC2 (5'-TAGTTTCTTTTCCTCCGCT-3') for T. canis, and Tcat1 (5'-GGAGAAGTAAACTC-3') and NC2 for $T$. cati [12]. The PCR amplification was carried out in $25 \mu \mathrm{l}$ reaction volumes containing $0.3 \mu \mathrm{l}$ $(5 \mathrm{u} / \mu \mathrm{l})$ of Taq DNA polymerase (Cinnagen, Iran), $2.5 \mu \mathrm{l}$ of $\times 10$ PCR buffer (Cinnagen, Iran), $0.9 \mu 1$ (50 mM) $\mathrm{MgCl}_{2}$ (Cinnagen, Iran), $0.5 \mu 1(10 \mathrm{mM})$ of deoxynucleotide triphosphate (dNTP) Mix (Cinnagen, Iran), 10-13 $\mu 1$ deionized distilled water, $1 \mu 1$ of each forward and reverse primers (15 pmol), 3-4 $\mu 1$ of bovine serum albumin $0.1 \%$ as enhancer, and 3-4 $\mu 1$ of DNA template. Amplifications were performed under the following conditions: Initial cycle at $94^{\circ} \mathrm{C}$ for $30 \mathrm{~s}$, followed by 30 cycles of denaturation at $94^{\circ} \mathrm{C}$ for $60 \mathrm{~s}$, annealing at $55^{\circ} \mathrm{C}$ for $30 \mathrm{~s}$ and extension by polymerase at $72^{\circ} \mathrm{C}$ for $30 \mathrm{~s}$ and a final cycle at $72^{\circ} \mathrm{C}$ 
for $5 \mathrm{~min}$. The PCR products were electrophoresized on $1 \%(\mathrm{~W} / \mathrm{V})$ agarose gel stained with DNA safe stain.

\section{LAMP assay}

The forward and backward external primers (F3 and B3) and forward and backward internal primers (FIP and BIP) of ITS2 gene were used to perform LAMP assay based on Macuhova et al. study [21]. The LAMP assay was conducted in $25 \mu 1$ of a reaction mixture consisting of $40 \mathrm{pmol} / \mathrm{uL}$ concentration of each inner primer (FIP and BIP), $5 \mathrm{pmol} / \mathrm{uL}$ concentration of each outer primer (F3 and B3), $8 \mathrm{U} B s t$ 2.0 DNA polymerase (New England Biolabs), $1 \mu 1$ SYBR Green I, $2.5 \mu 1 \times 10$ buffer, $1.4 \mathrm{mM}$ of dNTPs, $3 \mathrm{mM}$ of $\mathrm{MgSO}_{4}, 0.8 \mathrm{M}$ of betaine, and $1 \mu \mathrm{l}$ of template DNA. The mixture was incubated at $64^{\circ} \mathrm{C}$ for $60 \mathrm{~min}$ in a heating block and then heated at $80^{\circ} \mathrm{C}$ to terminate the reaction. A positive control of Toxocara DNA and water as a negative sample were included in each LAMP assay. At the end of incubation, the presence of the target gene was characterized by the presence of white turbidity of magnesium pyrophosphate which detected visually by the naked eye. Accuracy of the findings was confirmed by both electrophoresis and fluorescence detection. LAMP products were electrophoresized on $1.5 \%$ agarose gel and were observed under ultraviolet (UV) light after staining by safe stain for $30 \mathrm{~min}$. Positive samples showed the typical ladder pattern which was not a single band. For fluorescence detection, $1 \mu 1$ of SYBR Green I was added on LAMP products and were irradiated with a UV lamp and photographed. The presence of fluorescence indicated the presence of the target gene. To compare the analytical sensitivity between LAMP and PCR assays, the various numbers of Toxocara eggs (1-6 eggs/200 g soil) were evaluated.

\section{DNA sequencing, alignment and phylogenetic analysis}

To confirm the specificity of the LAMP primers, a single round PCR was done based on B3 and F3 outer primers. Amplicons of the ITS2 gene were purified with the Wizard SV Cleanup System (Promega). ABIPRISMTM 3130 Genetic Analyzer automated sequencer (Applied Biosystem, USA) directly sequenced PCR products from 10 randomly selected samples. Contigs (overlapped sequences) from all samples were aligned and edited at consensus positions compared to GenBank sequences of all regional species using Sequencher Tmv.4.1.4 Software for PC (Gene Codes Corporation). T. canis sequences of southwest Iran (Accession nos.; AB743614-AB743617 and AB819327-AB819330) were directly retrieved from GenBank database (FASTA format). The sequences pairwise distances (percent identity and divergence) between sequenced isolates and other country sequences were constructed using the MegAlign program from Laser Gene Bio computing Software Package (DNASTAR, Madison, WI). MEGA 5.05 software with maximum likelihood algorithm and Kimura2-parameter model were used in order to construct phylogeny tree. The diversity (haplotype and nucleotide diversity), neutrality indices (Tajima's D and Fu's Fs statistic) and fixation index $(F$ st $)$ were estimated by DnaSP software version 5.10 [24].

\section{Statistical analysis}

Statistical analysis was performed using SPSS version 16.0 software. The Student's t-test was used to compare the frequencies of Toxocara prevalence among city areas at a confidence interval (CI) of $95 \%$. The degree of agreement among diagnostic methods results was determined by Kappa ( $\kappa$ ) value with 95\% CIs.

\section{Results}

The findings of microscopy, PCR and LAMP methods are presented in Table-1.57 ( $\mathrm{n}=31.6 \%$ ) of 180 soil samples identified by microscopy which the highest and lowest prevalence rate were belonged to north $(21.1 \%)$ and east $(0 \%)$ regions, respectively. As well, $7.7 \%(\mathrm{n}=14)$ samples were dominantly diagnosed to $T$. canis by PCR method while no $T$. cati infection was found by PCR. The kappa coefficient between PCR and microscopy indicated a faint agreement (0.308) and this difference was not found to be statistically significant $(\mathrm{Pv}=0.745) .77(42.7 \%)$ of 180 soil samples were contaminated to T. cati $(\mathrm{n}=49: 27.2 \%)$, T. canis $(\mathrm{n}=28: 15.5 \%)$ (Table-1) and mixed infections ( $\mathrm{n}=22: 12.2 \%$, not shown in Table-1). The kappa

Table-1: Comparison of parasitological and molecular methods for the detection of Toxocara sp. eggs in different regions of Tabriz, northwest Iran.

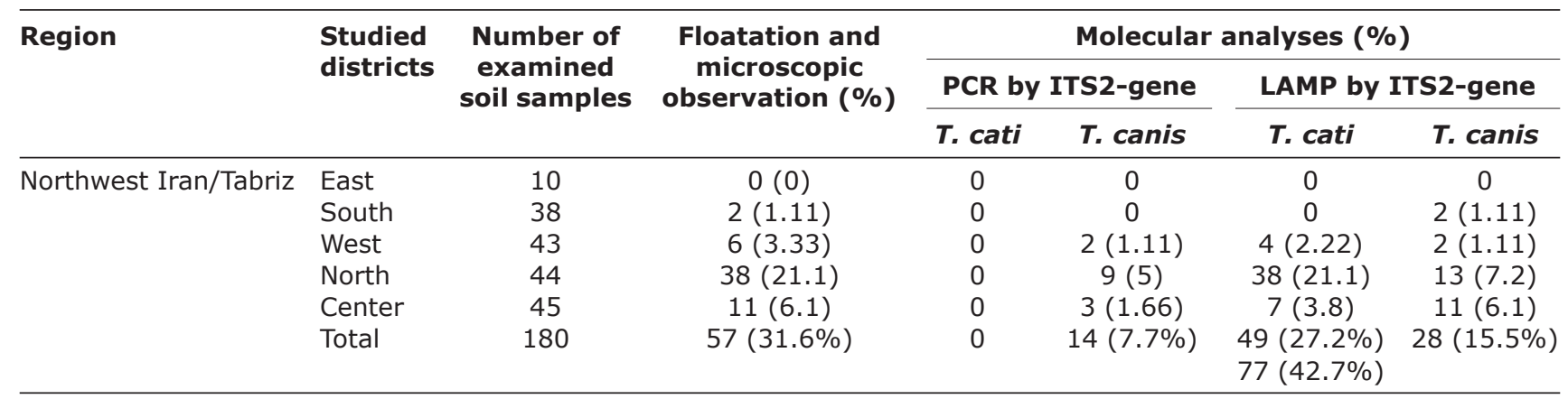

ITS=Internal transcribed spacer 2, PCR=Polymerase chain reaction, LAMP=Loop-mediated isothermal amplification 
coefficient between LAMP and microscopy indicated a strong agreement $(0.765, \mathrm{Pv}=0.01)$ but indicated a faint agreement among LAMP-PCR $(0.203$, $\mathrm{Pv}>0.05)$. The frequency of embryonated and unembryonated eggs recovered from streets and public parks is shown in Figure-1. The highest rate of fully embryonated eggs observed in the streets (13\%). The majority number of identified $T$. cati and $T$. canis eggs were ranged 1-3 and 1-10/200 g soil, respectively. The analytical sensitivity between LAMP and PCR assays were assessed based on number of Toxocara eggs/200 g soil (Figure-2). Findings show that PCR was able to distinguish more than 3 eggs/200 g soil (Figure-2a) while the LAMP detected 1-3 eggs/200 g soil sample (Figures-2b and $2 c$ ). Using species-specific primers, the PCR method showed $380 \mathrm{bp}$ fragment from T. canis (Figure-2a), however the $370 \mathrm{bp}$ fragment did not amplify for $T$. cati isolates. The frequency of Toxocara spp. identified by employed diagnostic methods is demonstrated in various divisions of Tabriz city according to Figure-3. Phylogenetic analysis revealed that the $T$. canis (AZE01-AZE05; deposited at the GenBank under accession numbers; KX181725-KX181729) grouped in its specific complex and Toxocara vitulorum (Accession number: KJ398347) was considered as an out-group branch (Figure-4). The percent identity (ranges: 96.5-100\%) and divergence (ranges: 0-5.6\%) among identified isolate (AZE02) and other countries' sequences shown in Figure-5. Multiple sequence alignment of $10 \mathrm{~T}$. canis isolates revealed two new haplotypes (AZE02 and AZE03) with low diversity values (haplotypes diversity; 0.295 , nucleotide diversity; 0.00088) and high neutrality values (Tajima's D; 0.71434 and Fu's Fs; 0.659 ) (Table-2). Fst as a degree of gene flow was generally low $(0.02156)$ between Toxocara populations of northwest and southwest Iran.

\section{Discussion}

In current inquiry, the high prevalence rate $(42.7 \%)$ of low Toxocara spp. loads was detected and identified by LAMP assay in soil samples of northwest Iran where despite the meaningful increasing sero-prevalence of Toxocara infection (3\% to $29.04 \%$ ) among young population [25-28] here is no inclusive examination on phylo-molecular epidemiology of infected soil samples yet. Up to now, several epidemiological surveys have been focused on contamination rate of public areas to Toxocara egg $[29,30]$. The global prevalence of Toxocara spp. in public parks estimated to be $9.75 \%, 6.73 \%, 28.31 \%, 11.57 \%$, $0.55 \%, 14.03 \%$, and $11.87 \%$ in North America, Latin America, Europe, Asia, Middle East, Australia, and Turkey, respectively $[29,30]$. Using zinc sulfate flotation technique, $31.6 \%$ of soil samples were contaminated with Toxocara eggs, which was higher than some formerly investigations accomplished in different sites of Iran. Epidemiological studies conducted in Iran demonstrate the contamination rate of public

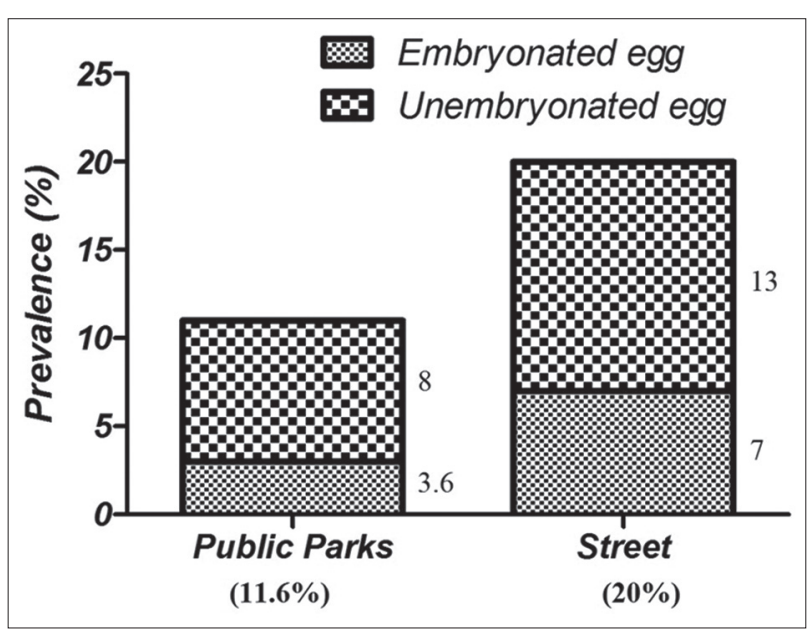

Figure-1: Prevalence of embryonated/unembryonated Toxocara eggs in the soil samples of streets and public parks recovered by the parasitological method.

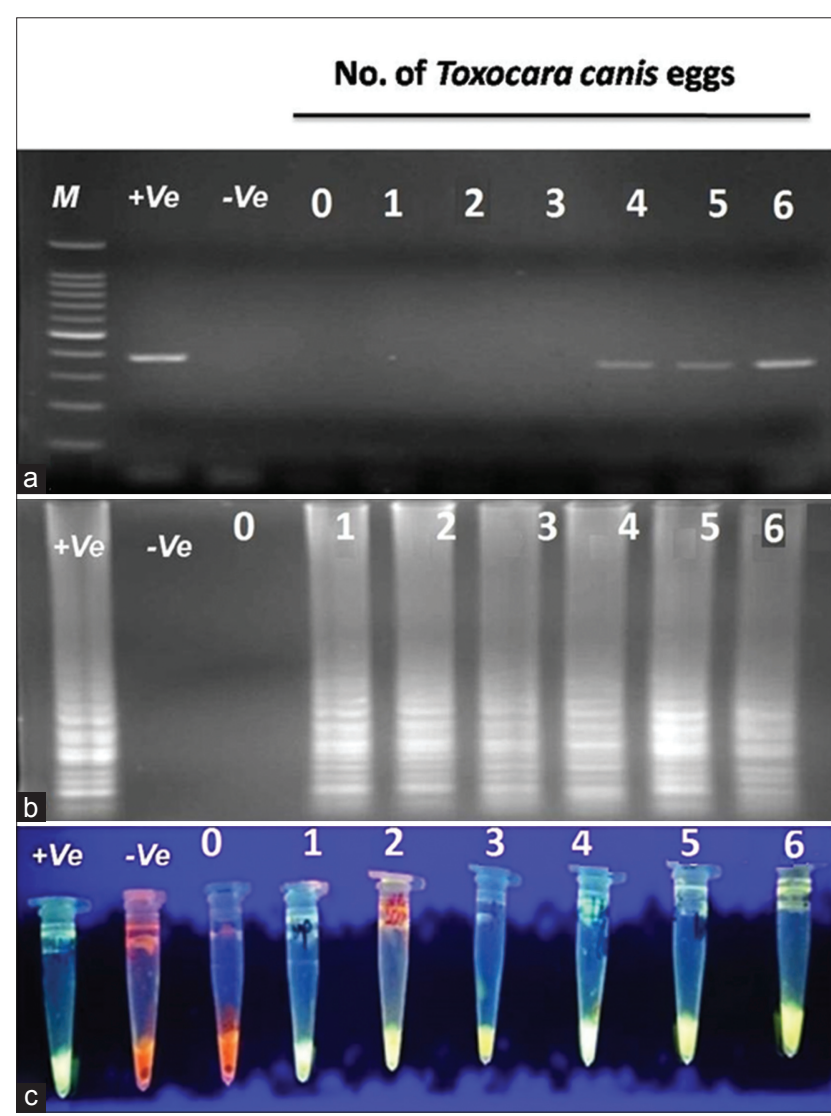

Figure-2: Sensitivity evaluation of loop-mediated isothermal amplification (LAMP) and polymerase chain reaction (PCR) assays based on number of Toxocara canis eggs in soil samples. (a) DNA amplification of Toxocara canis eggs by PCR on a $1.5 \%$ agarose gel (detection limit $>3$ eggs/200 g soil), (b) agarose gel electrophoresis of LAMP products (detection limit 1-3 eggs per $200 \mathrm{~g}$ soil), (c) LAMP, visual detection by fluorescence. $M=100$ bp DNA ladder marker; +Ve: Positive control; -Ve: Negative control (water).

parks has reported to be $6.3 \%, 3.9 \%, 30.4 \%$ and $10 \%$ in Shiraz (south), Urmia (northwest), Ahvaz (southwest) and Tehran (central) cities, respectively [22,31-33]. These differences may justify by several facts including socioeconomic status, geographical parameters, 


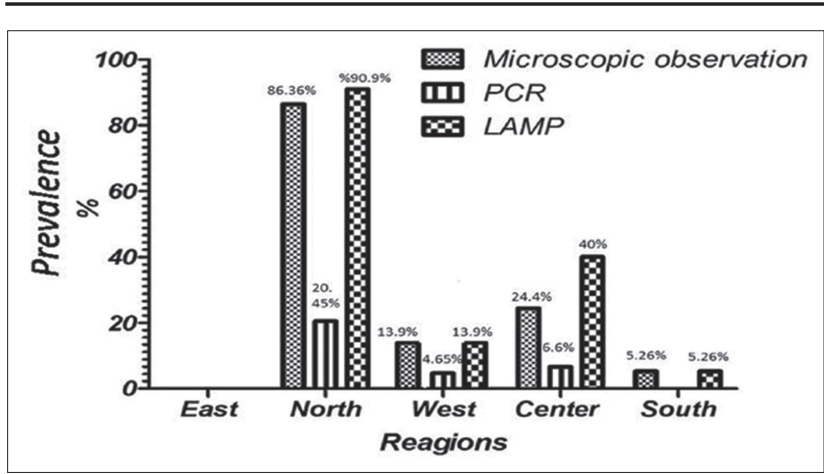

Figure-3: Prevalence of Toxocara spp. infection inferred by microscopic, polymerase chain reaction and loop-mediated isothermal amplification assays in various geographical regions of Tabriz.

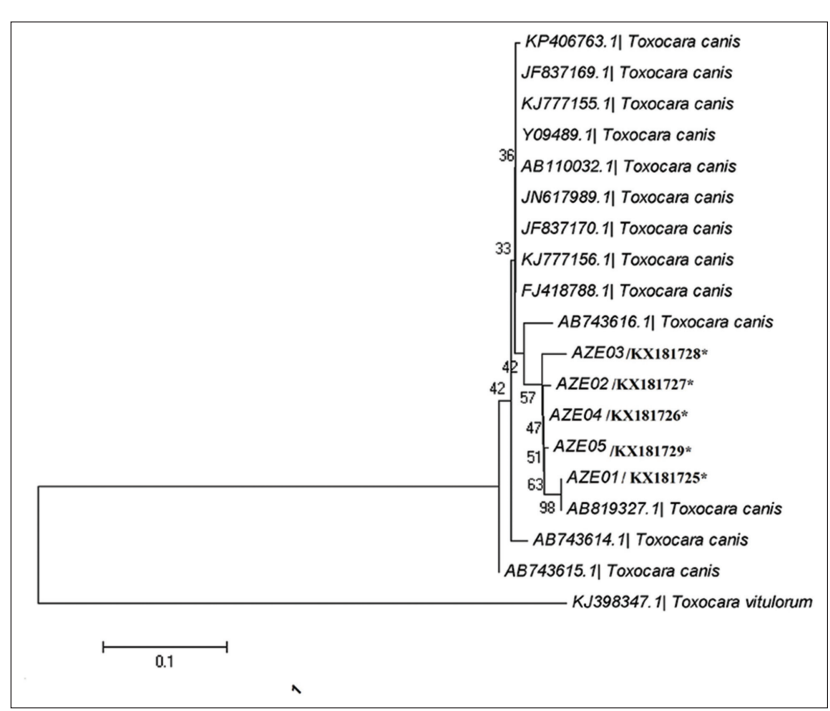

Figure-4: The phylogeny tree of Toxocara canis isolates according to the maximum-likelihood was conducted based on the multiple sequence alignment of internal transcribed spacer 2 gene by MEGA5.05. Distance represents the number of base substitutions per site. Toxocara vitulorum is the out-group branch.

poor sanitation, sample size, climatological variables, the number of stray cats and dogs, and especially employing various diagnostic tools. Furthermore, in this study, the majority of soil samples were collected from unfenced parks, which this could be provided the easy access of stray infected hosts.

A similar study conducted in Turkey has exhibited the high prevalence of soil contamination in unfenced public parks than fenced parks [30]. Hence, using antiparasitic drugs, enforcement of hygiene programs in park, management of stray dogs and cats, and increasing public awareness by municipal government can be decreased the prevalence of Toxocara infection.

In this study, the ratio of $T$. canis to $T$. cati was approximately found to be $1: 2(0.57)$ by the LAMP method, which is in conflict with other reports [22]. This shows that stray cats and dogs are the main contaminating animals in the region. However, our ongoing project on pet contamination showed that $11 \%$ of these animals are infected to T. canis (data not published yet). In this study, only $7.7 \%(n=14)$ of soil samples were diagnosed by PCR as $T$. canis (detection limit more than 3 eggs $/ 200 \mathrm{~g}$ ) while $54.9 \%$ of samples were diagnosed as $T$. cati $(27.2 \%)$ and $T$. canis $(15.5 \%)$ and mixed infections $(12.2 \%)$ by LAMP (detection limit 1-3 eggs/200 g). This shows that PCR is less sensitive than the LAMP assay. Theses discrepancies are described by some following facts: First, the activity of Taq DNA polymerase is inhibited by soil components such as humic acids, lipids, polysaccharides, and polyphenols/tannins while the Bst 2.0 DNA polymerase to overcome potential inhibitors in extracted DNA templates. Second, it is suggested that in a low Toxocara egg burden (particularly in T. cati egg), a considerable amount of parasite DNA is lost during the extraction and purification processes which in this case, a set of PCR primers cannot be specifically annealed and amplified target templates. They can interfere with the reaction at several levels, leading to different degrees of attenuation and even to complete inhibition [34].

Based on sequencing and phylogenetic findings, only two new haplotypes (AZE02 and AZE03, haplotype diversity: 0.295 , homology: $96.5-100 \%$ ) was identified among T. canis isolates. Low genetic diversity (HD; 0.295) of T. canis isolates can explain to be conserved the nature of ITS2 gene and its high copy number or small effective sample size [35]. In addition, this may be related to fertilization of Toxocara adult worm and/or to the longevity of the parasite in the stray dogs [36].

In this study, the $F$ st index was generally low (0.02156) among $T$. canis populations of northwest and southwest Iran. The statistically significant $F_{\text {st }}$ value indicates that the $T$. canis populations are not genetically well differentiated among mentioned regions. This shows that here is possibly an epidemiological drift due to transfer of alleles from one population to another population or vice versa.

\section{Conclusion}

The LAMP method because of its shorter reaction time, more sensitivity, simultaneous detection of environmental contamination and visual discriminatory of positivity to be appears as valuable alternative tool compared to PCR. Therefore, the detection of low Toxocara spp. loads from public district soils will help to expand epidemiological understanding of toxocariasis and employing preventive strategies in resource-limited endemic of Iran. Furthermore, Fst value in microevolutionary scale reflects new insights for further exploration in characterizing the local transmission patterns of Toxocara populations among various foci of Iran.

\section{Authors' Contributions}

$\mathrm{AS}$ and MO and TH: Contributed to the acquisition of data carried out the molecular genetic studies 


\begin{tabular}{|c|c|c|c|c|c|c|c|c|c|c|c|c|c|c|c|c|c|}
\hline \multicolumn{18}{|c|}{ Percent Identity } \\
\hline \multirow{16}{*}{ 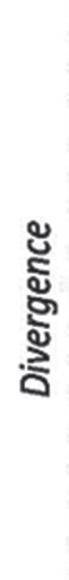 } & & 1 & 2 & 3 & 4 & 5 & 6 & 7 & 8 & 9 & 10 & 11 & 12 & 13 & 14 & & \multirow[b]{2}{*}{ AZE02 KX181727* } \\
\hline & 1 & & 94.4 & 95.6 & 96.5 & 100.0 & 96.2 & 96.8 & 96.8 & 96.8 & 96.8 & 96.8 & 87.6 & 96.8 & 96.8 & 1 & \\
\hline & 2 & 5.8 & & 95.0 & 97.4 & 94.4 & 97.6 & 97.6 & 97.6 & 97.6 & 97.6 & 97.6 & 89.4 & 97.6 & 97.6 & 2 & AB743614.1 Iran-Ahvaz \\
\hline & 3 & 4.6 & 5.2 & & 97.1 & 95.6 & 96.8 & 97.4 & 97.4 & 97.4 & 97.4 & 97.4 & 88.2 & 97.4 & 97.4 & 3 & AB743616.1 Iran-Ahvaz \\
\hline & 4 & 3.6 & 2.7 & 3.0 & & 96.5 & 99.1 & 99.7 & 99.7 & 99.7 & 99.7 & 99.7 & 90.6 & 99.7 & 99.7 & 4 & KP406763.1 Mexico \\
\hline & 5 & 0.0 & 5.8 & 4.6 & 3.6 & & 96.2 & 96.8 & 96.8 & 96.8 & 96.8 & 96.8 & 87.6 & 96.8 & 96.8 & 5 & AB819327.1 Iran-Ahvaz \\
\hline & 6 & 3.9 & 2.4 & 3.3 & 0.9 & 3.9 & & 99.4 & 99.4 & 99.4 & 99.4 & 99.4 & 90.3 & 99.4 & 99.4 & 6 & AB743615.1 Iran-Ahvaz \\
\hline & 7 & 3.3 & 2.4 & 2.7 & 0.3 & 3.3 & 0.6 & & 100.0 & 100.0 & 100.0 & 100.0 & 90.9 & 100.0 & 100.0 & 7 & FJ418788.1 Sri Lanka \\
\hline & 8 & 3.3 & 2.4 & 2.7 & 0.3 & 3.3 & 0.6 & 0.0 & & 100.0 & 100.0 & 100.0 & 90.9 & 100.0 & 100.0 & 8 & KJ777156.1 India \\
\hline & 9 & 3.3 & 2.4 & 2.7 & 0.3 & 3.3 & 0.6 & 0.0 & 0.0 & & 100.0 & 100.0 & 90.9 & 100.0 & 100.0 & 9 & JF837170.1 China \\
\hline & 10 & 3.3 & 2.4 & 2.7 & 0.3 & 3.3 & 0.6 & 0.0 & 0.0 & 0.0 & & 100.0 & 90.9 & 100.0 & 100.0 & 10 & JN617989.1 China \\
\hline & 11 & 3.3 & 2.4 & 2.7 & 0.3 & 3.3 & 0.6 & 0.0 & 0.0 & 0.0 & 0.0 & & 90.9 & 100.0 & 100.0 & 11 & AB110032.1 Japan \\
\hline & 12 & 3.6 & 1.6 & 3.0 & 0.3 & 3.6 & 0.7 & 0.0 & 0.0 & 0.0 & 0.0 & 0.0 & & 90.9 & 90.9 & 12 & Y09489.1 Australia \\
\hline & 13 & 3.3 & 2.4 & 2.7 & 0.3 & 3.3 & 0.6 & 0.0 & 0.0 & 0.0 & 0.0 & 0.0 & 0.0 & & 100.0 & 13 & KJ777155.1 India \\
\hline & 14 & 3.3 & 2.4 & 2.7 & 0.3 & 3.3 & 0.6 & 0.0 & 0.0 & 0.0 & 0.0 & 0.0 & 0.0 & 0.0 & & 14 & JF837169.1 China \\
\hline & & 1 & 2 & 3 & 4 & 5 & 6 & 7 & 8 & 9 & 10 & 11 & 12 & 13 & 14 & & \\
\hline
\end{tabular}

Figure-5: The percent of diversion and identity between the identified Toxocara canis (AZE02; KX181727*) and selected references' sequences circulating globally from GenBank database inferred by partial internal transcribed spacer 2 gene.

Table-2: Diversity and neutrality indices of $\boldsymbol{T}$. canis based on nucleotide sequences of ITS2-rDNA gene in northwest and southwest Iran.

\begin{tabular}{lccccccc}
\hline \multirow{2}{*}{ Region } & \multicolumn{4}{c}{ Diversity indices } & & \multicolumn{2}{c}{ Neutrality indices } \\
\cline { 2 - 5 } & $\mathbf{n}$ & Hn & Hd \pm SD & Nd $(\boldsymbol{\pi}) \pm$ SD & & Tajima's D & Fu's Fs statistic \\
\hline Northwest Iran (Tabriz) & 10 & 2 & $0.295 \pm 0.155$ & $0.00088 \pm 0.00077$ & & 0.71434 & 0.659 \\
Southwest Iran (Ahvaz) & 8 & 2 & $0.345 \pm 0.172$ & $0.00093 \pm 0.00088$ & & 0.69432 & 0.654 \\
\hline
\end{tabular}

ITS2=Internal transcribed spacer 2, $\mathrm{Hn}=$ Number of haplotypes, $\mathrm{Hd}=$ Haplotype diversity, $\mathrm{Nd}=$ Nucleotide diversity, T. canis=Toxocara canis, $\mathrm{SD}=$ Standard deviation

and have been involved in drafting the manuscript. ASh and AD and MMO: Participated in the design of the study, contributed to sample collection and helped to draft the manuscript. MA and PK and EA: Performed the statistical analysis and have been involved in critically revising the manuscript for important intellectual content. All authors read and approved the final version of the manuscript.

\section{Acknowledgments}

This study was financially supported by Tabriz Research Centre of Infectious and Tropical Diseases, Tabriz University of Medical Sciences, Tabriz, Iran. This article is derived from the master's thesis of the first author (Thesis No. 93/2-10/17).

\section{Competing Interests}

The authors declare that there is no competing interests.

\section{References}

1. Despommier, D. (2003) Toxocariasis: Clinical aspects, epidemiology, medical ecology, and molecular aspects. Clin. Microbiol. Rev., 16: 265-272.

2. Lee, A.C., Schantz, M., Kazacos, K.R., Montgomery, S.P. and Bowman, D.D. (2010) Epidemiologic and zoonotic aspects of ascarid infections in dogs and cats. Trends Parasitol., 26: 155-161.

3. Azian, M., Sakhone, L., Hakim, S.L., Yusri, M., Nurulsyamzawaty, Y., Zuhaizam, A., Rodi, I.M. and Maslawaty, M.N. (2008) Detection of helminth infections in dogs and soil contamination in rural and urban areas.
Southeast Asian. J. Trop. Med. Public Health, 39(2): 205-212.

4. Congdon, P. and Lloyd, P. (2011) Toxocara infection in the United States: The relevance of poverty, geography and demography as risk factors, and implications for estimating county prevalence. Int. J. Public Health, 56: 15-2.

5. Macpherson, C.N. (2005) Human behaviour and the epidemiology of parasitic zoonoses. Int. J. Parasitol., 35: 1319-1331

6. Smith, H., Holland, C., Taylor, M., Magnaval, J., Schantz, P. and Maizels, R. (2009) How common is human toxocariasis? Towards standardizing our knowledge. Trends Parasitol., 25: 182-188.

7. Gawor, J. and Borecka, A. (2004) The contamination of the environment with Toxocara eggs in Mazowieckie voivodship as a risk of toxocarosis in children. Wiad Parazytol., 50: 237-241.

8. Dubna, S., Langrova, I., Jankovska, I., Vadlejch, J., Peka, S., Napravnik, J. and Fechtner, J. (2007) Contamination of soil with Toxocara eggs in urban (Prague) and rural areas in the Czech Republic. Vet. Parasitol., 144: 81-86.

9. Glickman, L. and Shofer, F. (1987) Zoonotic visceral and ocular larva migrans. The veterinary clinics of North America. Small Anim. Pract., 17: 39.

10. Uga, S., Matsuo, J., Kimura, D., Rai, S.K. and Igarashi, K. (2000) Differentiation of Toxocara canis and T. cati eggs by light and scanning electron microscopy. Vet. Parasitol., 92: 287-294.

11. Borecka, A. (2004) Differentiation of Toxocara spp. eggs isolated from the soil by PCR-linked RFLP. Helminthologia, 41: 185-187.

12. Borecka, A. and Gawor, J. (2008) Modification of gDNA extraction from soil for PCR designed for the routine examination of soil samples contaminated with Toxocara spp. eggs. J. Helminthol., 82: 119-122.

13. Durant, J.F., Irenge, L.M., Fogt-Wyrwas, R., Dumont, C., 
Doucet, J.P., Mignon, B., Mignon, B., Losson, B. and Gala, J.L. (2012) Duplex quantitative real-time PCR assay for the detection and discrimination of the eggs of Toxocara canis and Toxocara cati (Nematoda, Ascaridoidea) in soil and fecal samples. Parasit. Vectors, 5(1): 288.

14. Nkouawa, A., Sako, Y., Nakao, M., Nakaya, K. and Ito, A. (2009) Loop-mediated isothermal amplification method for differentiation and rapid detection of Taenia species. J. Clin. Microbiol., 47(1): 168-174.

15. Kumagai, T., Furushima-Shimogawara, R., Ohmae, H., Wang, T.P., Lu, S., Chen, R., Wen, L. and Ohta, N. (2010) Detection of early and single infections of Schistosoma japonicum in the intermediate host snail, Oncomelania hupensis, by PCR and loop-mediated isothermal amplification (LAMP) assay. Am. J. Trop. Med. Hyg., 83(3): 542-548.

16. Liang, S.Y., Chan, Y.H., Hsia, K.T., Lee, J.L., Kuo, M.C., Hwa, K.Y., Chan, C.W., Chiang, T.Y., Chen, J.S., Wu, F.T. and Ji, D.D. (2009) Development of loop-mediated isothermal amplification assay for detection of Entamoeba histolytica. J. Clin. Microbiol., 47(6): 1892-1895.

17. Sotiriadou, I. and Karanis, P. (2008) Evaluation of loop-mediated isothermal amplification for detection of Toxoplasma gondii in water samples and comparative findings by polymerase chain reaction and immunofluorescence test (IFT). Diagn. Microbiol. Infect. Dis., 62(4): 357-365.

18. Poon, L.L., Wong, B.W., Ma, E.H., Chan, K.H., Chow, L.M., Abeyewickreme, W., Tangpukdee, N., Yuen, K.Y., Guan, Y., Looareesuwan, S. and Peiris, J.S. (2006) Sensitive and inexpensive molecular test for Falciparium malaria: Detecting Plasmodium falciparum DNA directly from heat-treated blood by loop-mediated isothermal amplification. Clin. Chem., 52: 303-306.

19. Nzelu, C.O., Gomez, E.A., Cáceres, A.G., Sakurai, T., Martini-Robles, L., Uezato, H., Mimori, T., Katakura, K., Hashiguchi, Y. and Kato, H. (2014) Development of a loop-mediated isothermal amplification method for rapid mass-screening of sand flies for Leishmania infection. Acta Trop., 132: 1-6.

20. Aonuma, H., Yoshimura, A., Perera, N., Shinzawa, N., Bando, H., Oshiro, S., Nelson, B., Fukumoto, S. and Kanuka, H. (2009) Loop-mediated isothermal amplification applied to filarial parasites detection in the mosquito vectors: Dirofilaria immitis as a study model. Parasit. Vectors, 2: 15.

21. Macuhova, K., Kumagai, T., Akao, N. and Ohta, N. (2010) Loop-mediated isothermal amplification assay for detection and discrimination of Toxocara canis and Toxocara cati eggs directly from sand samples. J. Parasitol., 96(6): 1224-1227.

22. Khademvatan, S., Abdizadeh, R. and Tavalla, M. (2014) Molecular characterization of Toxocara spp. from soil of public areas in Ahvaz Southwestern Iran. Act Trop., 135: 50-54.

23. Dada, B.J.O. (1979) A new technique for the recovery of Toxocara eggs from soil. J. Helminthol., 53: 141-144.
24. Rozas, J., Sanchez-DelBarrio, J.C., Messeguer, X. and Rozas, R. (2010) DnaSP, DNA polymorphism analyses by the coalescent and other methods. Bioinformatics, 19: 2496-2497.

25. Fallah, E., Mahami-Oskouei, M., Safaiyan, A., Mirsamadi, N., Hamzavi, F. and Mahami-Oskouei, L. (2012) The effect of keeping pet dogs and cats on toxocariasis. Yafteh, 13: 65-72.

26. Fallah, M., Azimi, A. and Taherkhani, H. (2003) Seroprevalence of toxocariasis in children aged 1-9 years in Western Islamic Republic of Iran. East Mediterr. Health J., 13: 1073-1077.

27. Fallah, E., Mahami-Oskouei, L., Mahami-Oskouei, M. and Safaiyan, A. (2013) Evaluation of indirect immunofluorescent antibody (IFA) and ELISA in diagnosis of visceral larva migranas. J. Ilam. Univ. Med. Sci., 21: 87-93.

28. Momeni, T., Mahami-Oskouei, M., Fallah, E., Safaiyan, A. and Mahami-Oskouei, L. (2016) Latent and asymptomatic Toxocara infection among young population in Northwest Iran: The necessity of informing people as a potential health risk. Scientifica, 2016: 3562056.

29. Avcioglu, H. and Burgu, A. (2008) Seasonal prevalence of Toxocara ova in soil samples from public parks in Ankara, Turkey. Vector Borne Zoonot. Dis., 8: 345-350.

30. Avcioglu, H. and Balkaya, İ. (2011) The relationship of public park accessibility to dogs to the presence of Toxocara species ova in the soil. Vector Borne Zoonot. Dis., 11(2): 177-180.

31. Motazedian, H., Mehrabani, D., Tabatabaee, S., Pakniat, A. and Tavalali, M. (2006) Prevalence of helminth ova in soil samples from public places in Shiraz. East Mediterr. Health J., 12: 562.

32. Tavassoli, M., Hadian, M., Charesaz, S. and Javadi, S. (2008) Toxocara spp. eggs in public parks of Urmia City, West Azerbaijan province Iran. Iran. J. Parasitol., 3(3): 24-29.

33. Khazan, H., Khazaei, M., Tabaee, S.S. and Mehrabi, A. (2012) Prevalence of Toxocara spp. eggs in public parks in Tehran City, Iran. Iran. J. Parasitol., 7(3): 38-42.

34. Moreira, D. (1998) Efficient removal of PCR inhibitors using agarose-embedded DNA preparations. Nuc. Acids Res., 26: 3309-3310.

35. Spotin, A., Gholami, S., Nasab, A.N., Fallah, E., Oskouei, M.M., Semnani, V., Shariatzadeh, S.A. and Shahbazi, A. (2015) Designing and conducting in silico analysis for identifying of Echinococcus spp. with discrimination of novel haplotypes: An approach to better understanding of parasite taxonomic. Parasitol. Res., 114(4): 1503-1509.

36. Spotin, A., Mahami-Oskouei, M., Harandi, M.F., Baratchian, M., Bordbar, A., Ahmadpour, E. and Ebrahimi, S. (2016) Genetic variability of Echinococcus granulosus complex in various geographical populations of Iran inferred by mitochondrial DNA sequences. Acta Trop. DOI: $10.1016 /$ j.actatropica.03.002. 